\title{
Recent advances in industrial carotenoid synthesis*
}

\author{
Hansgeorg Ernst \\ Fine Chemicals Research, GVF/B - B 9, BASF AG D-67056 Ludwigshafen, \\ Germany
}

Abstract: Symmetrical $\mathrm{C}_{40}$-carotenoids are efficiently produced by double Wittig olefination of the corrresponding $\mathrm{C}_{15}$-phosphonium salts with $\mathrm{C}_{10}$-dialdehyde. Industrial syntheses of lycopene-, astaxanthin-, and $\left(3 R, 3^{\prime} R\right)$-zeaxanthin- $\mathrm{C}_{15}$-phosphonium salts are discussed. An efficient route to a monoprotected $\mathrm{C}_{10}$-dialdehyde for the synthesis of unsymmetrical $\mathrm{C}_{40}$-carotenoids is presented. Primary polyene allyl alcohols can be converted to the corresponding aldehydes by "TEMPO" oxidation. A high-yield synthesis of meso-zeaxanthin as an example for syntheses of unsymmetrical carotenoids is presented.

\section{INTRODUCTION}

Eight out of the total of approximately 700 naturally occurring carotenoids are today produced synthetically on an industrial scale. These are the $\mathrm{C}_{40}$-carotenoids lycopene (1), $\beta, \beta$-carotene (2), $\left(3 R, 3^{\prime} R\right.$ )-zeaxanthin (3), canthaxanthin (4), and astaxanthin (5). These products are used as animal feed additives, in particular, in poultry farming and in aquaculture. $\beta, \beta$-Carotene is used for the direct coloring of foods, and lycopene, $B$-carotene, and zeaxanthin are employed as nutritional supplements.

In addition to these $\mathrm{C}_{40}$-carotenoids, three apocarotenoids are manufactured on an industrial scale. These are $B$-apo- $8^{\prime}$-carotenal (6), ethyl $B$-apo- $8^{\prime}$-carotenoate (7), and citranaxanthin $(\mathbf{8})$. The aldehyde is used as a food colorant while the ester and the ketone are employed as animal feed additives in poultry farming (Fig. 1).
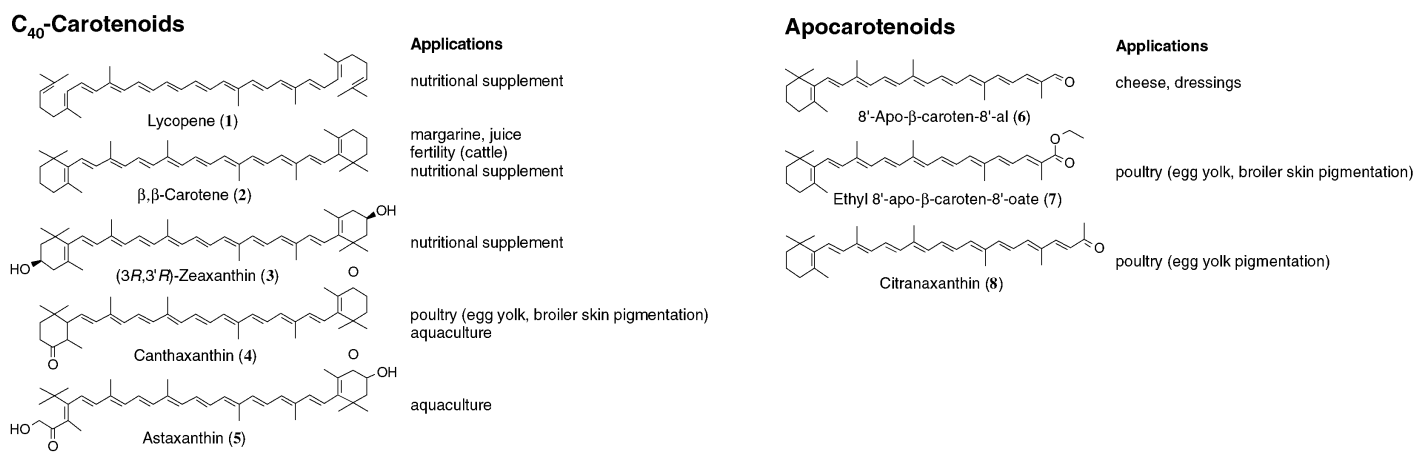

Fig. 1 Carotenoids by industrial synthesis.

\footnotetext{
*Lecture presented at the $13^{\text {th }}$ International Symposium on Carotenoids, Honolulu, Hawaii, USA, 6-11 January 2002 and originally published in Pure Appl. Chem. 74 (8), 1369-1382 (2002). In the original article, the figures were reproduced incorrectly; therefore, the article is being reprinted in its entirety.
} 
Apart from $\beta, \beta$-carotene (2) this entire range of products is manufactured exclusively by the companies Hoffmann-La Roche and BASF. Two further exceptions are that BASF is the only producer of the C33 ketone citranaxanthin (8) and Hoffmann-La Roche is-since 2001-the only producer of $\left(3 R, 3^{\prime} R\right)$-zeaxanthin $(\mathbf{3})$.

\section{INDUSTRIAL SYNTHESIS OF SYMMETRICAL CAROTENOIDS}

Without exception, the $\mathrm{C}_{40}$-carotenoids produced today by industrial synthesis have symmetrical structures, i.e., they have identical end-groups. The most efficient method for building up these symmetrical structures is the double Wittig condensation of a symmetrical $\mathrm{C}_{10}$-dialdehyde $\mathbf{9}$ as the central $\mathrm{C}_{10}$-building block with two equivalents of an appropriate $C_{15}$-phosphonium salt 10. In this process, in addition to the desired (all-E)-configured carotenoids, certain amounts of mono- and (di-Z)-stereoisomers of the newly formed disubstituted double bonds at $\mathrm{C}(11) / \mathrm{C}(12)$ and $\mathrm{C}\left(11^{\prime}\right) / \mathrm{C}\left(12^{\prime}\right)$ are produced. These mixtures of isomers are thermally isomerized as a rule, for example, by heating for several hours in heptane or ethanol, to form the desired (all- $E$ )-configured products. In doing so, the poorly soluble (all- $E$ )isomer crystallizes out and is thus removed from the isomerization equilibrium [1,2].

The productivity of this convergent synthesis strategy has been demonstrated in numerous symmetrical carotenoids. It is used industrially in production processes for lycopene (1), $\beta, \beta$-carotene (2), zeaxanthin (3), and astaxanthin (5) (Fig. 2).

The double Wittig olefination of $\mathbf{9}$ affords a highly efficient method capable of diverse application in the final stage of the industrial synthesis of carotenoids. Accordingly, the principal task in the development of industrial carotenoid syntheses consists in finding practical pathways to the corresponding $\mathrm{C}_{15}$-phosphonium salts which make technical and economic sense.

In doing this, there are fundamentally three obstacles to be surmounted.

1) The desired (E)-configurations must be established at trisubstituted $\mathrm{C}-\mathrm{C}$ double bonds which isomerize with difficulty; this task arises with lycopene (1).

2) Sensitive substitution templates are to be introduced at the end-groups. This problem must be solved for astaxanthin $(\mathbf{5})$.

3) The prerequisite for a synthesis of $\left(3 R, 3^{\prime} R\right)$-zeaxanthin $(3)$ is the introduction of stereogenic centers having a configuration identical to that in nature.

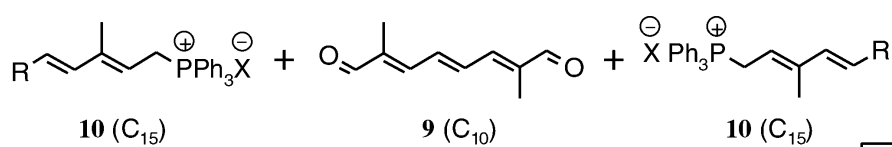<smiles>[R]C=CC(C)=CC=CC(C)=CC=CC(C)=CC=CC(C)=CC=CC=CC(C)=CC=CC(C)=CC=CC(C)=CC=CC(C)=CC=C</smiles>

\begin{tabular}{|l|l|}
\hline \multicolumn{1}{|c|}{} \\
\hline
\end{tabular}

Fig. $2 \mathrm{C}_{15}+\mathrm{C}_{10}+\mathrm{C}_{15}$-Strategy. 


\section{LYCOPENE}

The trisubstituted $\mathrm{C}-\mathrm{C}$ double bonds in the open-chain carotenoid lycopene 1 at positions (5)/(6) and $\left(5^{\prime}\right) /\left(6^{\prime}\right)$ isomerize from $(Z)$ to $(E)$ with difficulty [3]. In the synthetic method, these double bonds having the $(E)$-configuration must be introduced and retained so that the final product exhibits the desired high (all-E)-content.

The starting material for industrial lycopene syntheses is (E/Z)-pseudoionone $\mathbf{1 1}$ having $13 \mathrm{C}$ atoms; it is available in large quantities as the precursor in industrial vitamin A processes. From the mixture of $(E / Z)$-isomers, pure $(E)$-pseudoionone $(E)$-11 is isolated by distillation.

Extension by two $\mathrm{C}$ atoms to form the $\mathrm{C}_{15}$-building block (E)-vinyl pseudoionol $\mathbf{1 2}$ can be done in a two-step sequence consisting of ethynylation to $\mathbf{1 3}$ and subsequent partial hydrogenation. In the BASF process 12 is obtained in one step by the reaction of pseudoionone with a vinyl Grignard reagent.

The key step in the synthesis of lycopene is the rearrangement of $(E)$-vinyl pseudoionol $\mathbf{1 2}$ to form the lycopene $\mathrm{C}_{15}$-phosphonium salt. At the same time, the tendency of $(Z)$-isomers to form at the trisubstituted $\mathrm{C}(5) / \mathrm{C}(6)$ and $\mathrm{C}\left(5^{\prime}\right) / \mathrm{C}\left(6^{\prime}\right)$ double bonds in the polyene chain must be suppressed as far as possible.

Various solutions have been developed for this task in the synthesis.

According to a process described in the patent literature, $\mathbf{1 2}$ is treated with a mixture of triphenylphosphane and acetic acid to produce the $\mathrm{C}_{15}$-phosphonium acetate in high yield but with an (E/Z)-selectivity of only 2.3:1. Enrichment of the (all-E)-isomer by anion exchange of acetate for chloride $\mathbf{1 4}$ is described. (Z)-isomers of $\mathbf{1 4}$ can be depleted by crystallization. In this way, the (E/Z)-isomer ratio in the mother liquor can be raised to a value of 3.7:1 (Fig. 3) [4].

To increase the (all- $E$ )-content of the $\mathrm{C}_{15}$-phosphonium salt we conducted an extensive screening of the reaction conditions. In doing this, it emerged that stereoselectivity can be raised to a value of 3.7:1 if triphenylphosphonium sulfonates (e.g., methanesulfonate) are used. Analysis of this process by

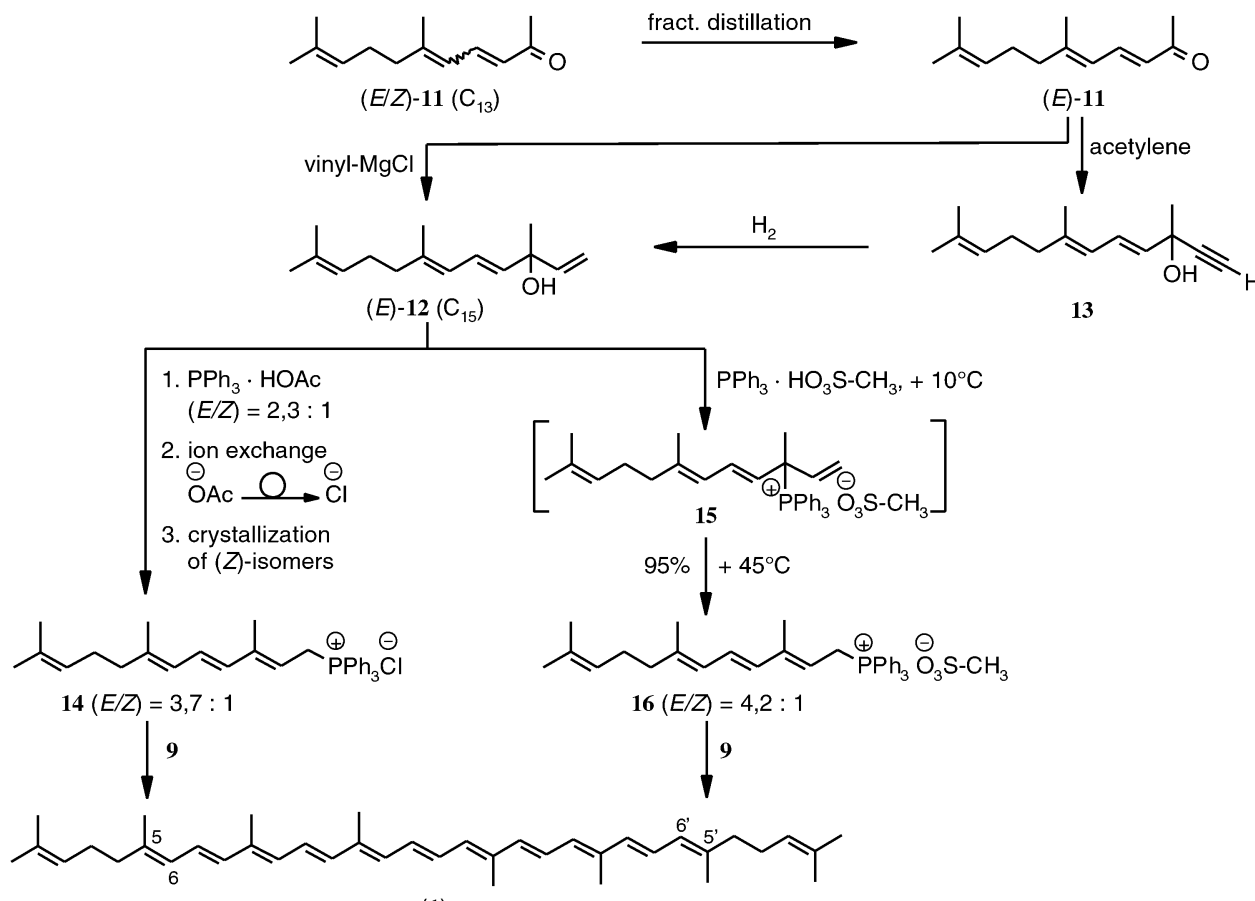

(1)

Fig. 3 Industrial synthesis of lycopene (1).

(C) 2002 IUPAC, Pure and Applied Chemistry 74, 2213-2226

[replaces Pure Appl. Chem. 74 (8), 1369-1382 (2002)] 
means of NMR indicates that it passes through an intermediate tertiary phosphonium salt 15. In a second step, 15 rearranges at elevated temperature into the desired primary phosphonium salt 16. In doing so, the isomerization of the critical double bond can be largely suppressed. By careful temperature control, the (E/Z)-selectivity of the process can be raised to a ratio of 4.2:1. The yield of $\mathbf{1 6}$ is $95 \%$ [5]. 16 without further enrichment can react in solution under classic Wittig conditions with 9 to form lycopene (1) having a high (all-E) content (Fig. 3).

\section{SYNTHESES OF XANTHOPHYLLS}

A strategy for the synthesis of xanthophylls, in which the ketoisophorone $\mathbf{1 7}$ is employed as a common precursor, was developed by Hoffmann-La Roche. From this synthetic $\mathrm{C}_{9}$-building block, which is readily available from petrochemical base materials, the substitution template of the finished product in question is introduced by simple reactions in sometimes shielded or protected form. The cyclic $\mathrm{C}_{9}$-ketones react by various routes to form the corresponding $\mathrm{C}_{15}$-phosphonium salts. The classic double Wittig olefination using $\mathrm{C}_{10}$-dialdehyde 9 yields the $\mathrm{C}_{40}$-xanthophylls. This synthetic strategy has been described at length in reviews [1,6]. Industrial processes for astaxanthin and zeaxanthin have been elaborated.

\section{ASTAXANTHIN}

The $\alpha$-hydroxy group of astaxanthin (5) is introduced at the stage of the $\mathrm{C}_{9}$-ketoisophorone building block 17 in protected and shielded form via the following four steps:

- $\quad$ epoxidation of the $\mathrm{C}-\mathrm{C}$ double bond to yield $\mathbf{1 8}$

- rearrangement of the epoxide to the sodium enolate $\mathbf{1 9}$ of the corresponding tricarbonyl compound

- catalytic hydrogenation of the reactive carbonyl group to $\mathbf{2 0}$

- $\quad$ fixing and protection of the enolized $\alpha$-hydroxyketone group as a dioxolane

Here the process described by Hoffmann-LaRoche proceeds via a five-membered ketal ring 21 [7] while BASF decided in favor of a five-membered acetal ring 22 produced by twofold acid-catalyzed addition of the diol to a vinyl ether (Fig. 4) [8].

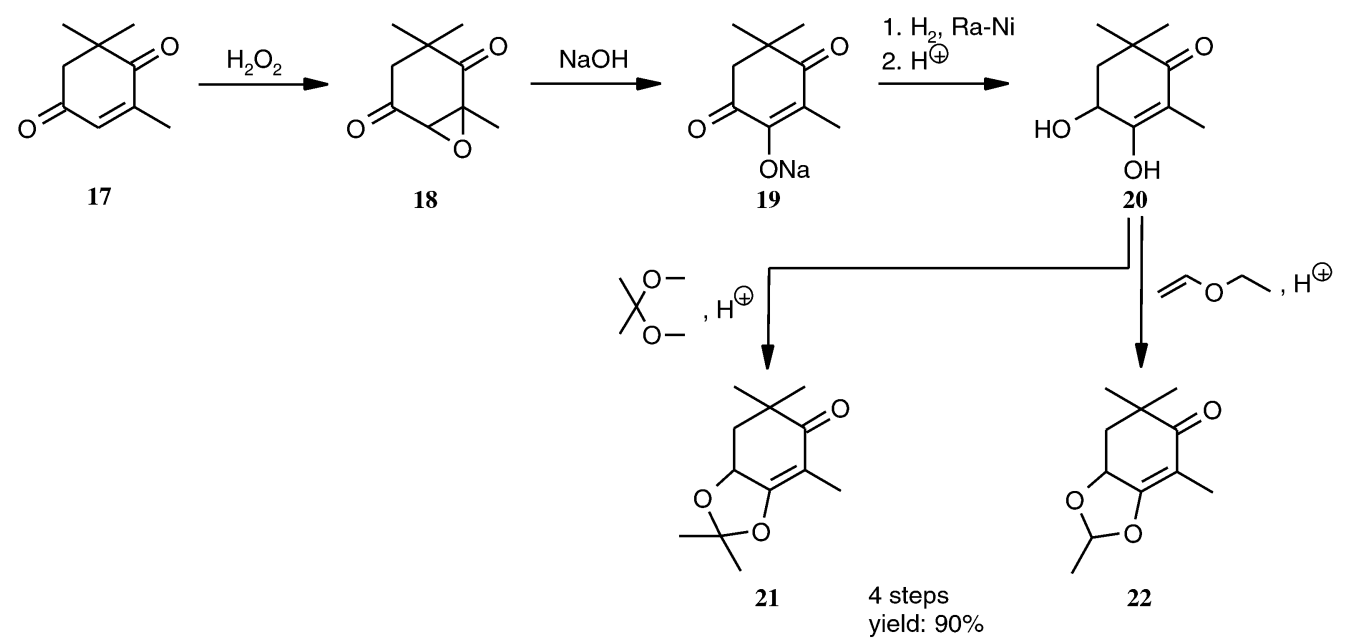

Fig. 4 Cyclic $\mathrm{C}_{9}$-units for astaxanthin (5). 
In the variant synthesis BASF has worked out, the protected $\mathrm{C}_{9}$-building block $\mathbf{2 2}$ is converted to astaxanthin in five stages. First of all, the keto group is allowed to react with the lithium acetylide $\mathbf{2 3}$ of the $\mathrm{C}_{6}$-vinylbutynol building block protected as an acetal to yield the $\mathrm{C}_{15}$-intermediate $\mathbf{2 4}$. After elimination of water, cleavage of protective groups, and partial reduction of the triple bond to a double bond, the $\mathrm{C}_{15}$-building block $\mathbf{2 5}$ having the substitution pattern of astaxanthin is obtained. Successive reaction with hydrobromic acid and triphenylphosphane yields the $\mathrm{C}_{15}$-phosphonium salt $\mathbf{2 6}$. In the classic double Wittig olefination using dialdehyde $\mathbf{9}$, astaxanthin (5) is obtained in over $80 \%$ yield (Fig. 5) [8].

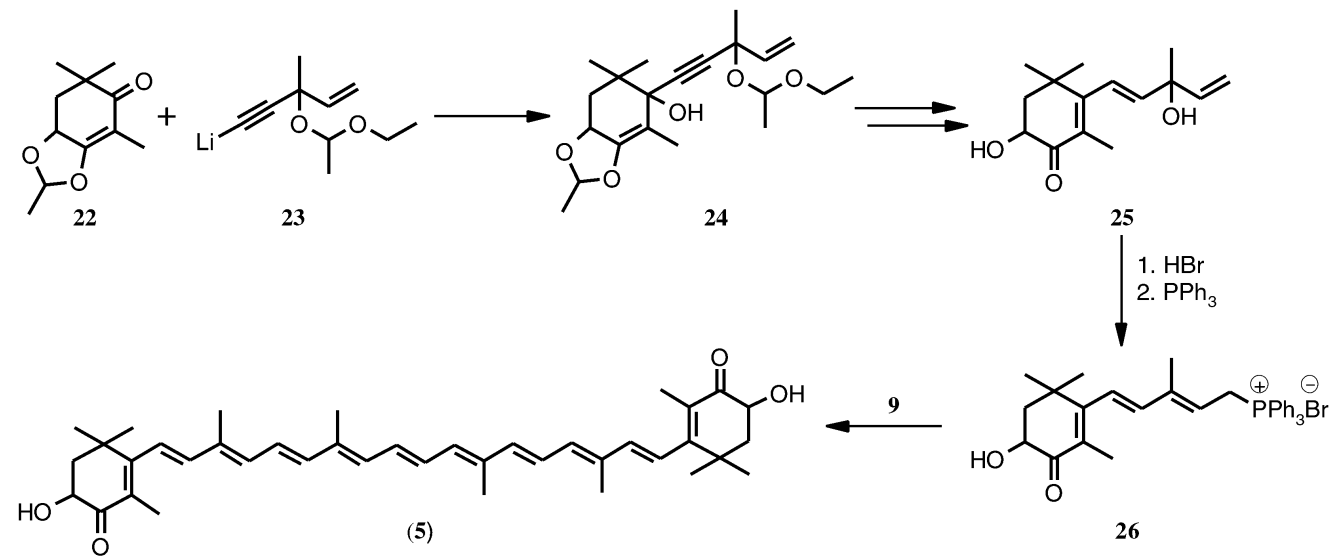

Fig. $5 \mathrm{C}_{15}$-Phosphonium salt 26 and final step.

\section{(3R,3R)-ZEAXANTHIN}

\section{Enantiopure $\mathrm{C}_{9}$-synthons: $(S)$-phorenol and $(4 R, 6 R)$-actinol}

Natural zeaxanthin $(3)$ occurs mainly in the $\left(3 R, 3^{\prime} R\right)$-configuration. Accordingly, the principal task in a synthesis of (3) consists in introducing the chiral center in a configuration identical to that in nature. (3) is the first enantiopure carotenoid produced by total industrial synthesis. First of all an enantiopure $\mathrm{C}_{9}$-hydroxyketone has to be produced. For this purpose, Hoffmann-LaRoche authors have described both an efficient chemical process - an enantioselective catalytic hydrogenation-as well as a combination of a biocatalytic process-a yeast reduction —with a chemical reduction.
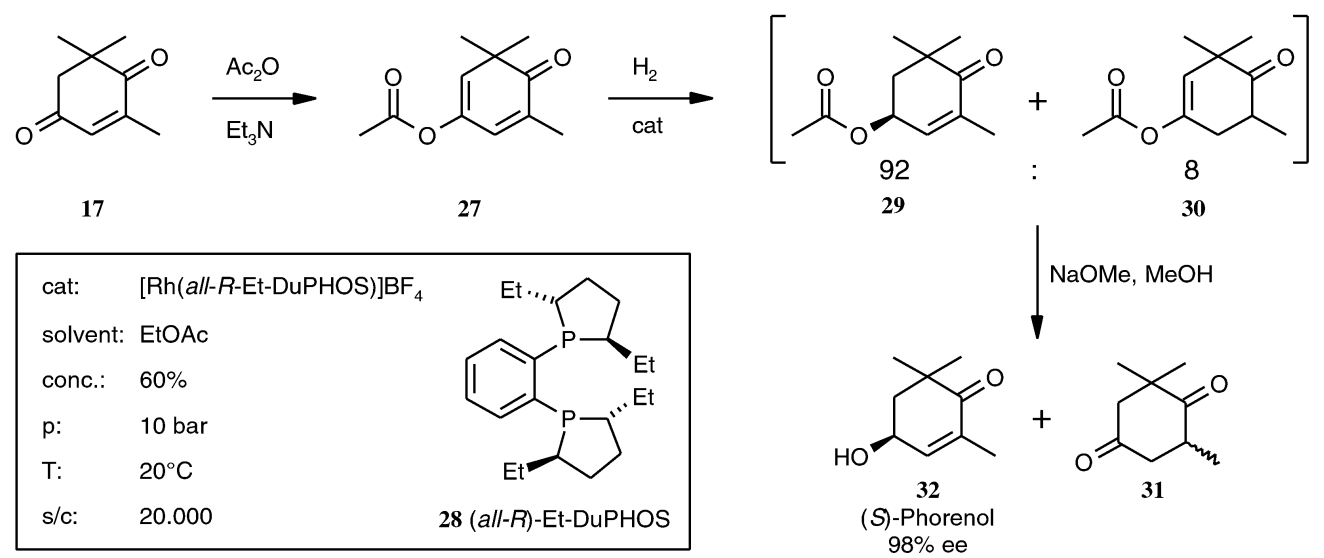

29

30

Fig. 6 (S)-Phorenol 32.

(C) 2002 IUPAC, Pure and Applied Chemistry 74, 2213-2226

[replaces Pure Appl. Chem. 74 (8), 1369-1382 (2002)] 
The enol acetate $\mathbf{2 7}$ of ketoisophorone $\mathbf{1 7}$ is employed as substrate for the enantioselective catalytic hydrogenation. Known cationic rhodium diphosphine complexes, for example, (all-R)-EtDuPhOS 28, are used as the hydrogenation catalysts. Apart from the outstanding ee values, the high concentration and favorable substrate/catalyst ratio are noteworthy. In quantitative reactions, a regioselectivity in hydrogenation of $92 \%$ to the desired $\mathbf{2 9}$ has been published. After methanolysis of the acetyl group and separation off of the racemic saturated diketone $\mathbf{3 1}$ as by-product, $(S)$-phorenol $\mathbf{3 2}$ in $98 \%$ ee is isolated (Fig. 6) $[9,10]$.

32 was converted into the $(R)$-configured $\mathrm{C}_{15}$-phosphonium salt $\mathbf{3 3}$ in a complex reaction sequence via subsequent chain lengthening by $\mathrm{C} 1-, \mathrm{C} 3-$, and $\mathrm{C} 2$-units $[11,12]$; the yields insofar as they have been published are moderate. Accordingly, despite its elegant pathway to the enantiopure $\mathrm{C}_{9}$-building block 32, this zeaxanthin synthesis has scarcely any industrial relevance.

As an alternative to the enantioselective hydrogenation, the stereogenic center can be introduced in a biocatalytic process. Hoffmann-La Roche have developed a method for the enantioselective reduction of the $\mathrm{C}-\mathrm{C}$ double bond into the $(6 R)$-configured levodione $\mathbf{3 4}$ by means of baker's yeast. This stereocenter controls the subsequent catalytic hydrogenation of the sterically less-shielded carbonyl group so that the 4-hydroxyketone $\mathbf{3 5}$ having the $(4 R, 6 R)$-configuration is obtained in approximately the ratio of 4:1 relative to its ( $4 S)$-epimer 36 [13]. The main product can be separated from its epimer by distillation using a process described by BASF (Fig. 7) [14].
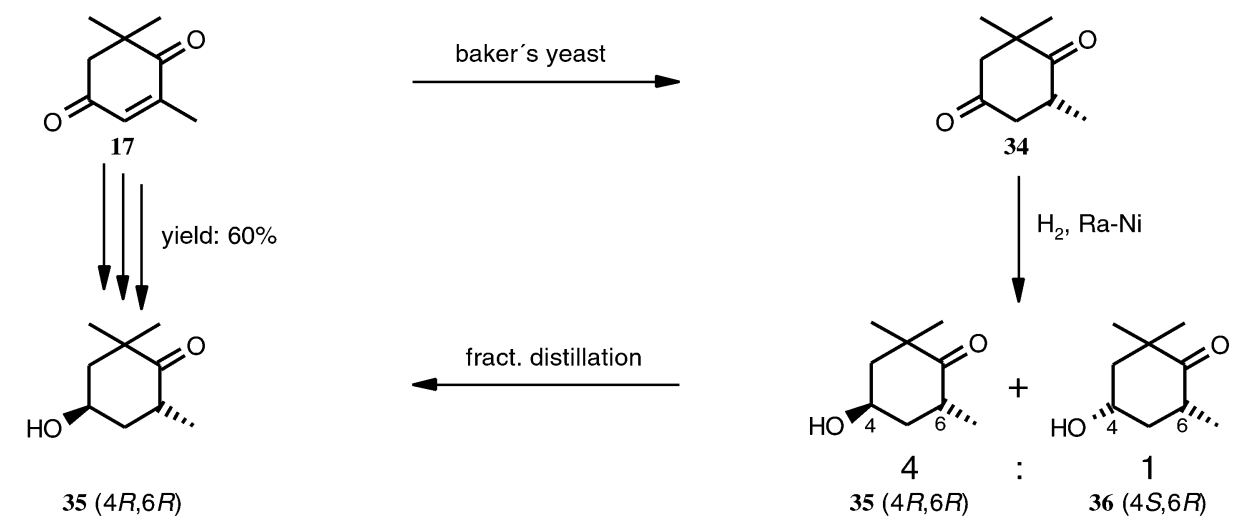

Fig. $7(4 R, 6 R)$-Actinol 35.

This process exhibits two weaknesses:

- The biocatalyst must be separated from the useful product and disposed of.

- The stereoselectivity of the catalytic hydrogenation does not come up to the requirements imposed on an industrial method.

These weak points resulted in substantial improvements in the process, which are reported in new patent applications filed by Hoffmann-La Roche. Various species of yeast bonded onto photopolymerized resins are described for the biocatalytic enantioselective hydrogenation of the $\mathrm{C}-\mathrm{C}$ double bond. Immobilization allows simple separation and recirculation of the biocatalyst. A $(R)$-levodione $\mathbf{3 4}$ yield of $70 \%$ at a purity of over $99 \%$ is reported for the pilot-plant scale [15].

The reduction of $\mathbf{3 4}$ to $\mathbf{3 5}$ via transfer hydrogenation proceeds with high stereoselectivity. Ruthenium sulfonamide amino complexes, for example, 37, are employed as catalysts while isopropanol serves as hydrogen donor. The coproduct acetone is continuously removed from the reaction mixture by distillation. The stereoselectivity is $94 \%$. After crystallization, 35 having an ee value of more than $99 \%$ is obtained (Fig. 8) [16]. 


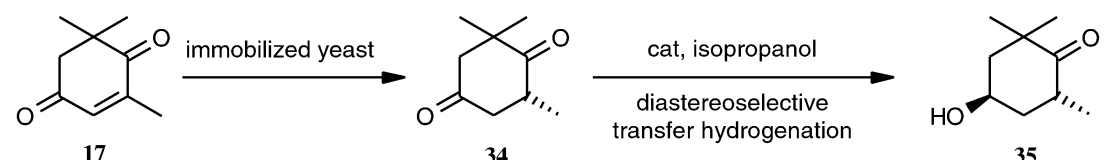

stereoselectivity $94 \%$ crystallized yield $75 \%$

purity $>99 \%$

ee $>99,5 \%$

\begin{tabular}{|lll|}
\hline cat: & [RuH (Ts-en\{-H\})(p-Cym)] \\
solvent: & isopropanol \\
conc.: & $10 \%$ \\
$\mathrm{~T}:$ & $40^{\circ} \mathrm{C}$ \\
s/c: & 1.000 & 37 \\
\hline
\end{tabular}

Fig. $8(4 R, 6 R)$-Actinol 35: recent improvements.

\section{$\mathrm{C}_{15}$-Phosphonium salt from $(4 R, 6 R)$-actinol- $\mathrm{C}_{9}+\mathrm{C}_{2}+\mathrm{C}_{4}$-strategy}

Starting from 35 , various synthesis strategies have been developed for building up to the $\mathrm{C}_{15}$-phosphonium salt [17-19]. Hoffmann-LaRoche authors have described a process that is based on stepwise extension by $\mathrm{C}_{2}$ - and a $\mathrm{C}_{4}$-building block. Acetylene serves as the $\mathrm{C}_{2}$-building block and methyl vinyl ketone is used as the $\mathrm{C}_{4}$-building block [18]. Despite an excellent overall yield this synthesis is not without problems from the point of view of industrial implementation. A protective group must be introduced and then cleaved off again on three occasions: first of all, an acetal protective group which is stable under basic conditions for the ethynylation $(\mathbf{3 5} \rightarrow \mathbf{3 8})$, then an acid-resistant protective group for the copper sulfate-catalyzed dehydration $(\mathbf{3 8} \rightarrow \mathbf{3 9})$ and then a protective group which is stable to bases once more for the introduction of the $\mathrm{C}_{4}$-building block and the hydride reduction of the resultant propargyl alcohol $(\mathbf{3 9} \rightarrow \mathbf{4 0})$. This laborious chemistry using protective groups is unsatisfactory in both economic and ecological terms (Fig. 9).

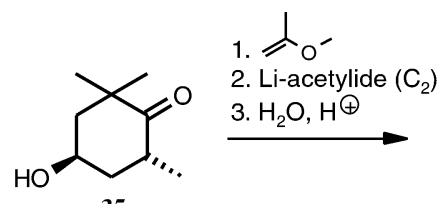

35

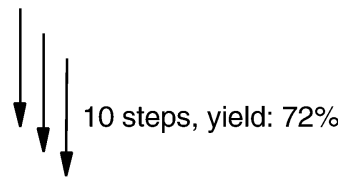<smiles>CC1=C(/C=C/C(C)=C/C[Pb](Cl)(Cl)Cl)C(C)(C)C[C@@H](O)C1</smiles>

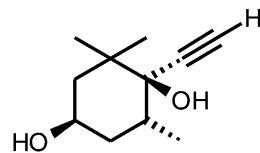

38

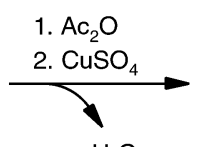

$-\mathrm{H}_{2} \mathrm{O}$<smiles>CC#CC1=C(C)C[C@@H](OC(C)=O)CC1(C)C</smiles>

39<smiles>C=CC(C)(O)/C=C/C1=C(C)C[C@@H](OC(C)(C)OC)CC1(C)C</smiles>

40

Fig. $9 \mathrm{C}_{15}$-Phosphonium chloride 33 from $(4 R, 6 R)$-actinol 35. 


\section{$C_{15}$-Phosphonium salt from $(4 R, 6 R)$-actinol- $C_{9}+C_{1}+C_{3}+C_{2}$-strategy}

Accordingly, we have developed a synthesis which avoids these disadvantages. The basic idea consists in protecting the secondary hydroxy group on $\mathrm{C}(3)$ of the zeaxanthin in the form of a cyclic ether in the $\mathrm{C}_{9}$-building block itself, and in devising the following stages in such a way that it is necessary only to provide protection against basic reaction conditions. The key step in this approach to the synthesis is the addition of dichloromethyl lithium as a $\mathrm{C}_{1}$-building block. Dichloromethyl lithium is produced in situ by the reaction of dichloromethane with butyl lithium. The free secondary hydroxy group is deprotonated to the lithium alkoxide by the reagent used in excess.

During the addition of the dichloromethyl lithium to the keto group, a chlorooxirane $\mathbf{4 1}$ is first of all obtained as intermediate. By increasing the reaction temperature, the epoxide ring is opened by intramolecular attack of the alkoxide and it then rearranges itself to the aldehyde $\mathbf{4 2}$ with elimination of lithium chloride. The successive extensions, first of all by $\mathrm{C}_{3}$ and then by $\mathrm{C}_{2}$, to form $\mathbf{4 3}$ take place in the usual way by aldol condensation with acetone followed by addition of vinyl Grignard reagent. Reaction of the latter with triphenylphosphane hydrobromide causes opening of the oxabicycloheptane system to yield the $\mathrm{C}_{15}$-phosphonium bromide $\mathbf{4 4}$ (Fig. 10) [19].
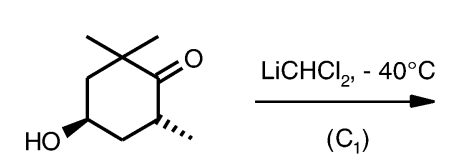

$\left(C_{1}\right)$

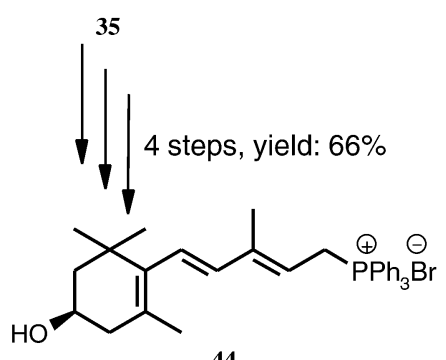

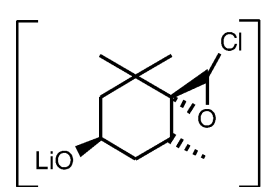

41

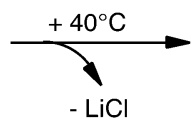

$-\mathrm{LiCl}$

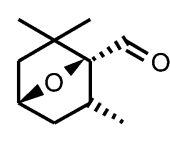

42

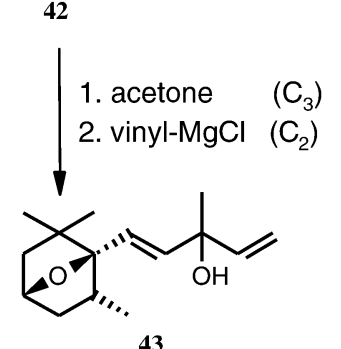

Fig. $10 \mathrm{C}_{15}$-Phosphonium bromide 44 via bicyclic aldehyde $\mathbf{4 2}$.

\section{UNSYMMETRICAL $\mathrm{C}_{40}$-CAROTENOIDS}

The five $\mathrm{C}_{40}$-carotenoids, which are synthesized today on an industrial scale, are exclusively symmetrical carotenoids, that is to say, the structures have identical end-groups. A glance into the "Key to Carotenoids" shows, however, that the great majority of $\mathrm{C}_{40}$-carotenoids have unsymmetrical structures, i.e., the end-groups differ in chemical structure or in configuration [20]. These carotenoids include substances such as $\alpha$-carotene, $\beta$-cryptoxanthin, and also lutein (45), which have long aroused great interest on account of their physiological properties. On account of the different absolute configurations at its chiral centers $\left(3 R, 3^{\prime} S\right)$-meso-zeaxanthin $(46)$ is an unsymmetrical carotenoid from this point of view.

\section{(3R,3'S)-meso-zeaxanthin}

\section{Isomerization of lutein}

We were interested in access to high-yield methods for the preparation of chemically and stereochemically highly pure (46). The only route described in the literature for obtaining prepared quantities of 
<smiles>CC1=C[C@H](O)CC(C)(C)[C@H]1/C=C/C(C)=C/C=C/C(C)=C/C=C/C=C(C)/C=C/C=C(C)/C=C/C1=C(C)C[C@@H](O)CC1(C)C</smiles>

Fig. 11 Isomerization of lutein (45).

this xanthophyll is a partial synthesis, that is to say, the base-catalyzed isomerization of lutein (45), which for its part is isolated from natural sources (Fig. 11).

The isomerization of lutein, (45) to meso-zeaxanthin (46) is described in various patents [21-23]. As a general principle in these cases, (45) is heated for a relatively long time in the presence of a strong base. This isomerization reaction does not proceed to completion, however. Due to the elimination of water under the strongly basic reaction conditions, anhydrous secondary products are formed. Lutein from natural sources is usually accompanied by $\left(3 R, 3^{\prime} R\right)$-zeaxanthin (3). If a chemically and sterically highly pure meso-zeaxanthin (46) is to be obtained from this process, isomerization must be followed by an extremely costly ultrapurification stage. This partial synthetic pathway did not appear suitable to us for the preparation of (46) on a preparative scale in the quality we required. Total chemical synthesis was, accordingly, the route of choice.

\section{Synthesis of unsymmetrical $C_{40}$-carotenoids: $C_{15}+C_{10}+C_{15}$-strategy}

If it is intended to synthesize unsymmetrical carotenoids uncontaminated by the corresponding symmetrical products on the basis of the $\mathrm{C}_{15}+\mathrm{C}_{10}+\mathrm{C}_{15}$ synthesis strategy without costly purification of

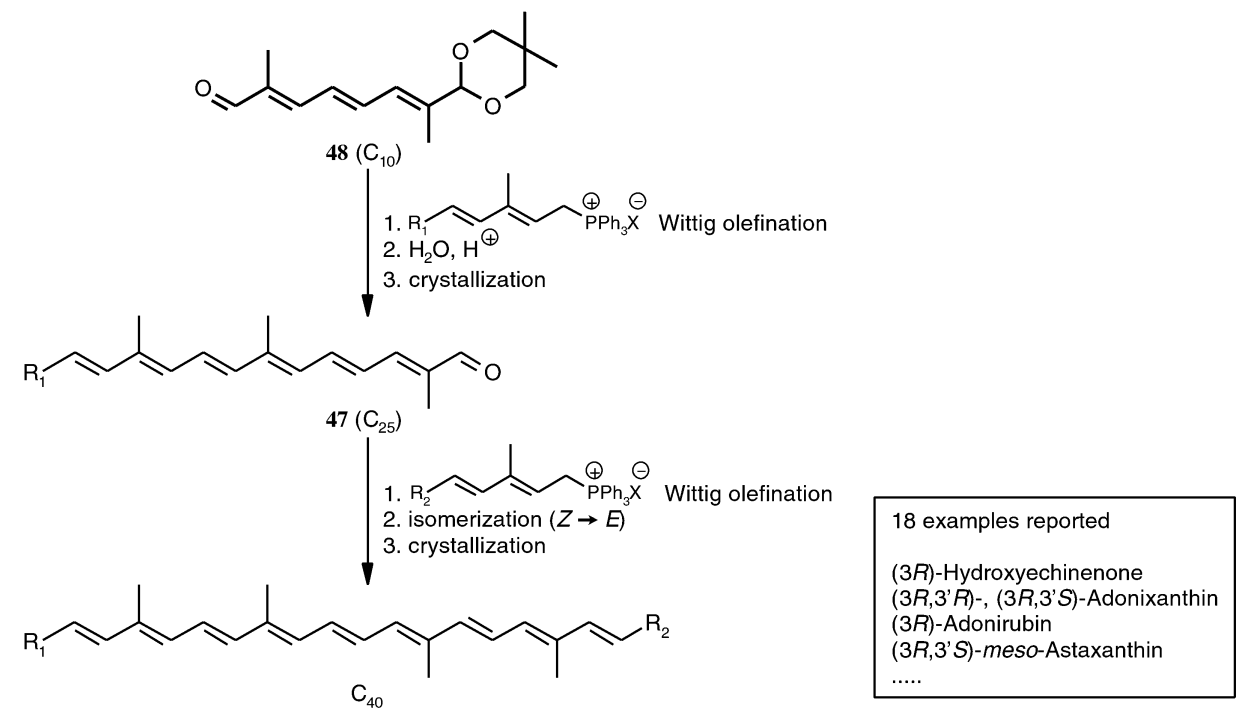

Fig. 12 Synthesis of unsymmetrical $\mathrm{C}_{40}$-carotenoids via $\mathrm{C}_{10}$-dialdehyde monoacetal 48 .

(C) 2002 IUPAC, Pure and Applied Chemistry 74, 2213-2226

[replaces Pure Appl. Chem. 74 (8), 1369-1382 (2002)] 
the $\mathrm{C}_{25}$-intermediates 47 , the Wittig reactions of the central $\mathrm{C}_{10}$-building block with the two phosphonium salts must proceed to completion selectively one after the other. This selectivity needed for the synthesis of a homogeneous product is ensured only when a $\mathrm{C}_{10}$-dialdehyde is employed in which one carbonyl group is protected in the form of an acetal 48. In polyene synthesis, cyclic acetals, especially dimethyl-1,3-dioxan, are usually used on grounds of stability. This approach was developed by Hoffmann-LaRoche authors and confirmed by the synthesis of a series of $B$-apo-11'-carotenals and unsymmetrical $\mathrm{C}_{40}$-carotenoids, including meso-astaxanthin (Fig. 12) [24].

\section{Directed synthesis of $\mathrm{C}_{10}$-dialdehyde monoacetal from $\mathrm{C}_{5}$-building blocks}

The only synthesis disclosed in the literature for the production of a monoacetal of the $\mathrm{C}_{10}$-dialdehyde consists in the selective hydrolysis of the corresponding diacetal by brief contact with hydrochloric acid under carefully controlled reaction conditions. The pure monoacetal is obtained only after expensive purification in a reported yield of only $37 \%$ [24]. This pathway to $\mathrm{C}_{10}$-dialdehyde monoacetal has proved to be unsuitable as a basis for the efficient synthesis of unsymmetrical carotenoids.

One of the basic tools of polyene chemistry in BASF is a construction set made up of $\mathrm{C}_{5}$-building blocks. On looking into this construction set we found two molecules which appeared to be suitable for the targeted and selective build-up of the mono-protected $\mathrm{C}_{10}$-dialdehyde. These were the $\mathrm{C}_{5}$-ester phosphonate 49 and the $\mathrm{C}_{5}$-acetal aldehyde 50. At BASF, various production processes have been worked out for these intermediate products [25].

In the first step, the $\mathrm{C}_{5}$-ester phosphonate $\mathbf{4 8}$ is allowed to react with the $\mathrm{C}_{5}$-acetal aldehyde $\mathbf{5 0}$ under classic Wittig-Horner conditions. The yield is just about quantitative. The crude olefination product $\mathbf{5 1}$ can be purified by continuous distillation in high vacuum. In the second step, the ester is reduced to the alcohol using vitride in toluene. The crude alcohol $\mathbf{5 2}$ is oxidized to the aldehyde $\mathbf{4 8}$ in the presence of the "TEMPO"/copper(I) chloride catalyst system by molecular oxygen as oxidizing agent. Following crystallization, very pure $\mathbf{4 8}$ totally uncontaminated by free dialdehyde is obtained. The overall yield of crystalline product from the entire sequence is an impressive $78 \%$ (Fig. 13).

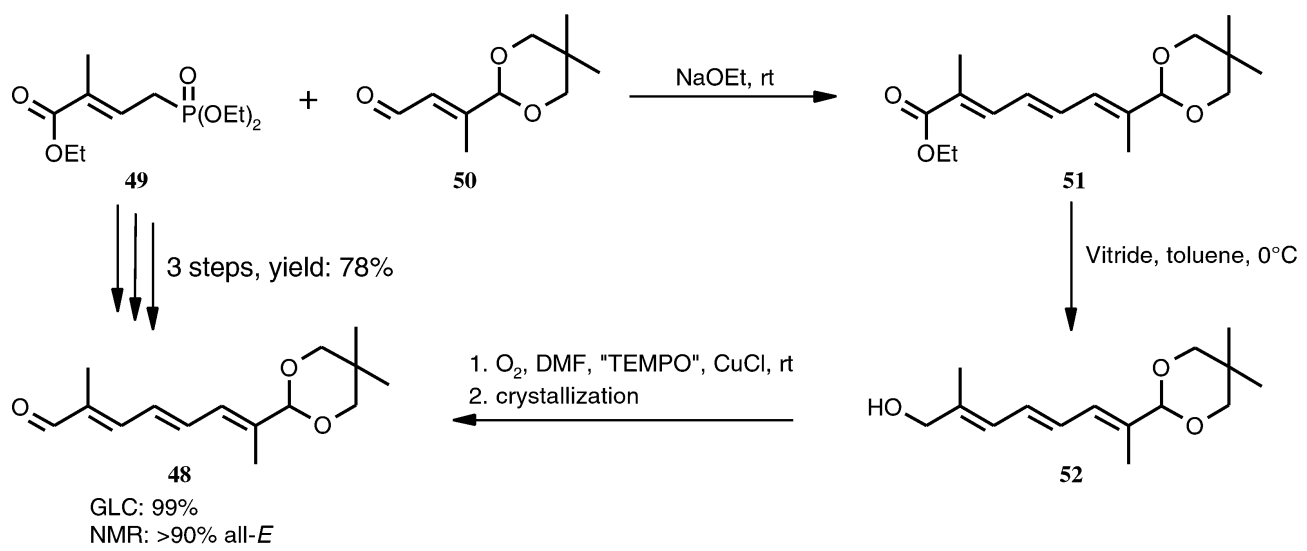

Fig. $13 \mathrm{C}_{10}$-Dialdehyde monoacetal 48 from $\mathrm{C}_{5}$-building blocks 49 and $\mathbf{5 0}$.

\section{“TEMPO"-oxidations of polyene allyl alcohols}

The oxidation of primary polyene allyl alcohols to the corresponding aldehydes had long lacked a satisfactory practical solution. As a rule, the oxidizing agent, manganese dioxide or chromium trioxide complexes, for instance, was employed in stoichiometric quantity or even in excess. However, these methods have no industrial relevance. We have picked up on a method which had been published by 
Semmelhack in 1984, namely, the oxidation by nitrosium ions of primary allyl and benzyl alcohols using molecular oxygen in the presence of copper(I) chloride. The source of nitrosium ions is the commercially available tetramethylpiperidine- $N$-oxyl 53, "TEMPO" for short. The variant described by Semmelhack using copper(I) chloride/oxygen afforded very good yields when applied under mild conditions to simple primary allyl and benzyl alcohols without overoxidation or double bond isomerization being observed [26].

We have used this method on a series of primary polyene allyl alcohols. Although molecular oxygen is used, this method has proved to be widely applicable to the synthesis of polyene aldehydes. Some of the yields are excellent. $\alpha$-Branched allyl alcohols $(\mathbf{5 2}, \mathbf{5 5})$ can be oxidized without difficulty. The oxidation of (all-E)-retinol $\mathbf{5 8}$ is doubtless a particularly impressive example. In this case, crystalline (all-E)-retinal is obtained in a yield of $90 \%$. Even the reaction of allyl alcohol 55, having five openchain conjugated double bonds, proceeds satisfactorily in high yield (Fig. 14).

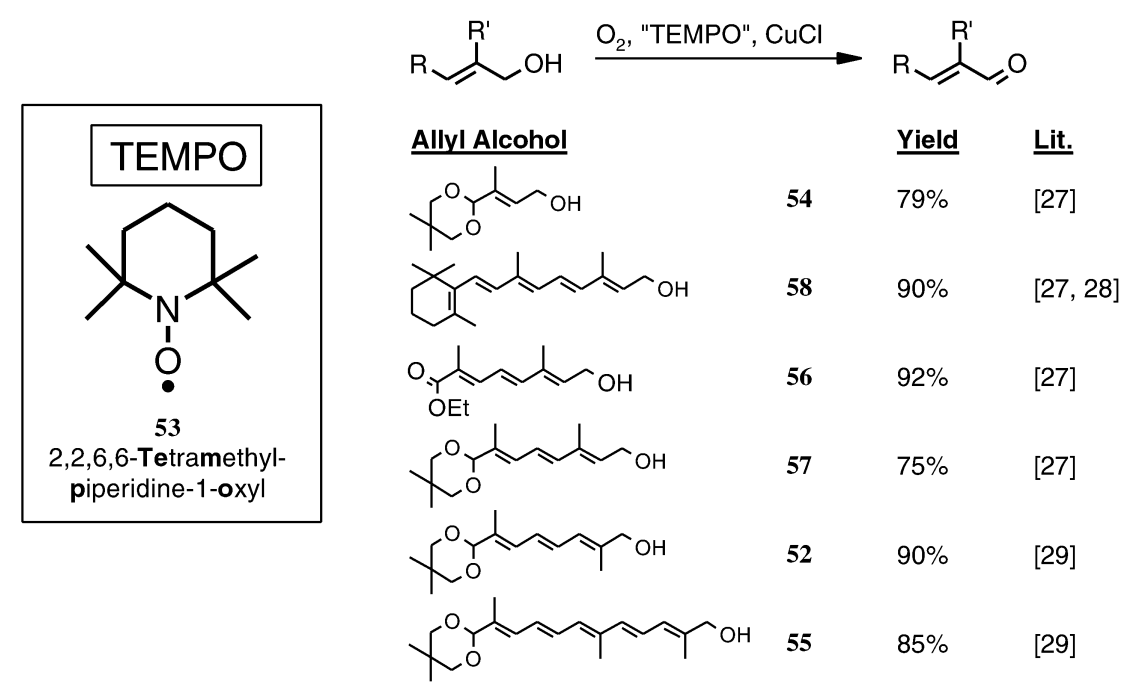

Fig. 14 "TEMPO"-oxidations of polyene allyl alcohols.

\section{Enantiopure $\mathrm{C}_{11}$-building blocks}

The next task on the synthetic pathway to meso-zeaxanthin (46) consisted in providing both enantiomers of a suitable precursor on a laboratory scale in approximately equal quantities and at the highest level of enantiomeric purity. In this case, the literature to date has concentrated substantially on precursors of $\left(3 R, 3^{\prime} R\right)$-zeaxanthin (3). If equal quantities of both enantiomers of one synthetic building block are needed, the idea of a classic racemate resolution soon arises. In the case of zeaxanthin precursors, the possibility of deriving diastereomeric intermediates from the secondary hydroxy group, (e.g., esters) presents itself. The racemic mixture of diastereopure $\mathrm{C}_{11}$-acetylene diol 59, which can be obtained from racemic trans-actinol via three stages by analogy with the $(R, R)$-zeaxanthin synthesis discussed above, proved to be a particularly suitable candidate for racemate resolution. Derivatization ensued completely selectively at the secondary hydroxy group. In terms of its chemistry and configuration, this zeaxanthin precursor was completely stable to the conditions that are needed to introduce and cleave off a chiral resolving reagent.

The resolving reagent of choice proved to be $(R)$-2,4-dichlorophenoxypropionic acid $\mathbf{6 0}$ ("2,4-DCPP-acid") which is available in BASF as an intermediate from the field of crop protection agents. By crystallization of the diastereomeric esters $\mathbf{6 1}$ of the $\mathrm{C}_{11}$-building block using this optically pure carboxylic acid, the diastereomeric ester belonging to the " $(R)$-series" is enriched and can be 
obtained in highly purified form by recrystallization. The diastereomeric ester of the " $(S)$-series" is correspondingly enriched in the mother liquor. After saponification of the esters, the optically $\mathrm{C}_{11}$-building blocks 38 [“( $R)$-series”] respectively $\mathbf{6 2}$ [“( $S)$-series”] are isolated by crystallization.

Thus using one resolving reagent, both enantiomers are obtained in equal quantities. By recirculation of the mother liquor and reesterification, the racemate can be completely resolved into the enantiomers without loss. The resolving agent can be also recycled by simple acid/base-separation. The optical purity of both enantiomerically pure building blocks $(\mathbf{3 8}, \mathbf{6 2})$ was determined by means of GC analysis on chiral columns and corresponded to over $99 \%$ ee (Fig. 15).

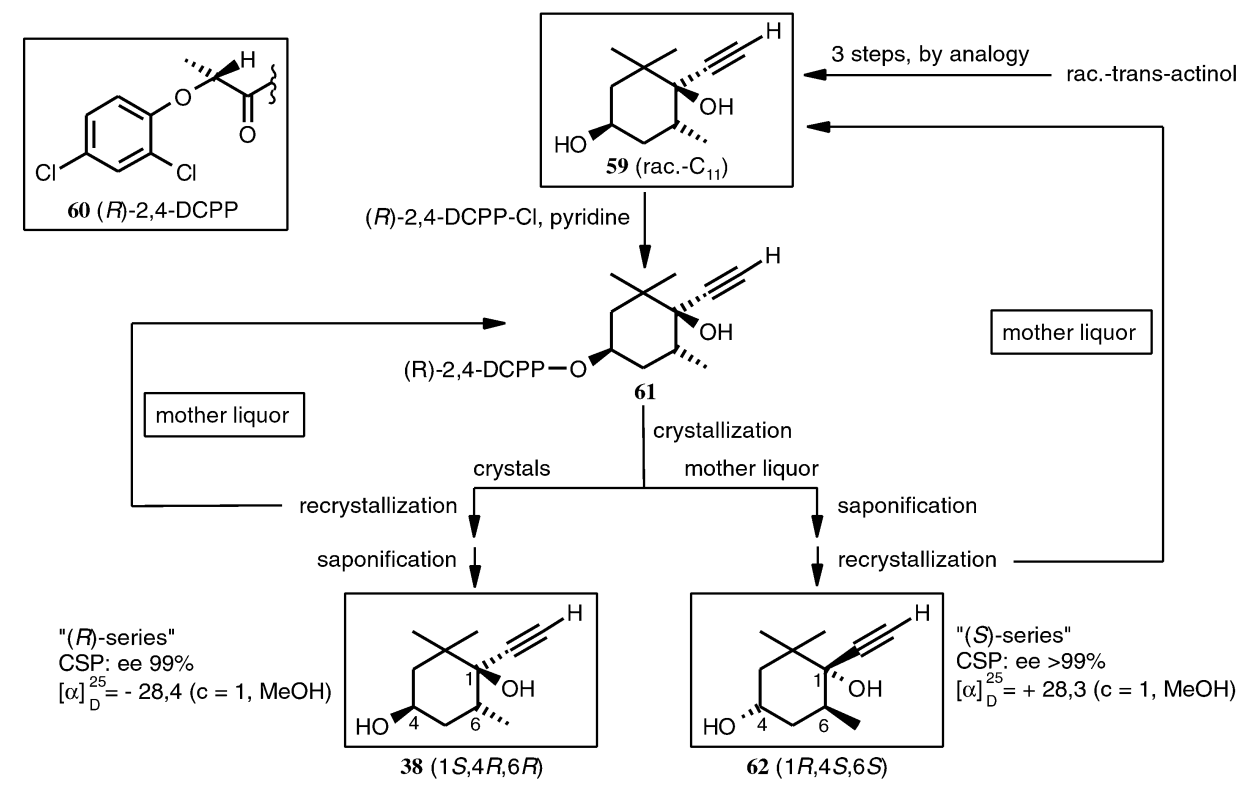

Fig. 15 Enantiopure $\mathrm{C}_{11}$-building blocks.

\section{(S)- $\mathrm{C}_{15}$-Phosphonium salt}

By analogy with the process disclosed in the literature for the " $(R)$-series" [17], we additionally converted the $\mathrm{C}_{11}$-building block $\mathbf{6 2}$ of the $(S)$-series into the $(S)$ - $\mathrm{C}_{15}$-phosphonium salt $\mathbf{6 3}$. The interme-

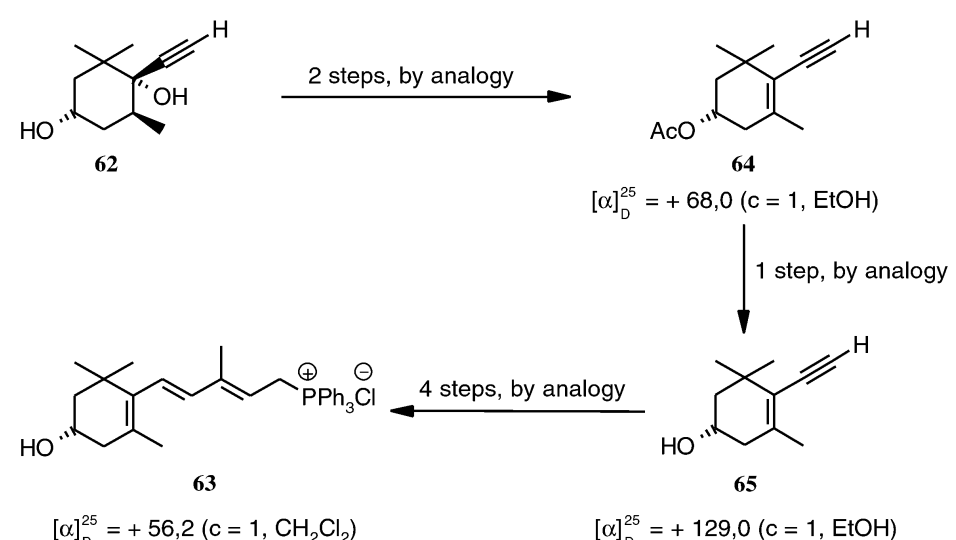

Fig. 16 Enantiopure $(S)-\mathrm{C}_{15}$-phosphonium salt 63. 
diate products 64 and 65 in the $(S)$-series had not yet been described. The measured angles of rotation are in good agreement with the values published for the $(R)$-series (Fig. 16).

\section{FINAL STEPS}

In the final steps of the synthesis of meso-zeaxanthin (46), the enantiopure $\mathrm{C}_{15}$-phosphonium salts are condensed with the central $\mathrm{C}_{10}$-building block $\mathbf{4 8}$ in successive Wittig reactions. A decisive factor for the stereochemical purity of the finished product was the completeness of reaction of the first Wittig reaction since purification of the $\mathrm{C}_{25}$-intermediate was to be avoided. In a simple sequence, comprising heating of the $(R)$-configured phosphonium salt 33 (in slight excess) with 48 in butylene oxide/ethanol, acid-catalyzed cleavage of acetal and heating of the resultant crude $\mathrm{C}_{25}$-aldehyde with $(S)$-configured phosphonium salt 63, we obtained pure meso-zeaxanthin (46) in an overall yield of $79 \%$ by crystallization from ethanol. The chemical purity of (46) as determined by HPLC was $98 \%$. Stereochemical purity was determined by a method described in the literature [30]. It was more than $99 \%$ for mesozeaxanthin with less than $0.3 \%$ in each case for $(R, R)$ - and $(S, S)$-enantiomers (Fig. 17).

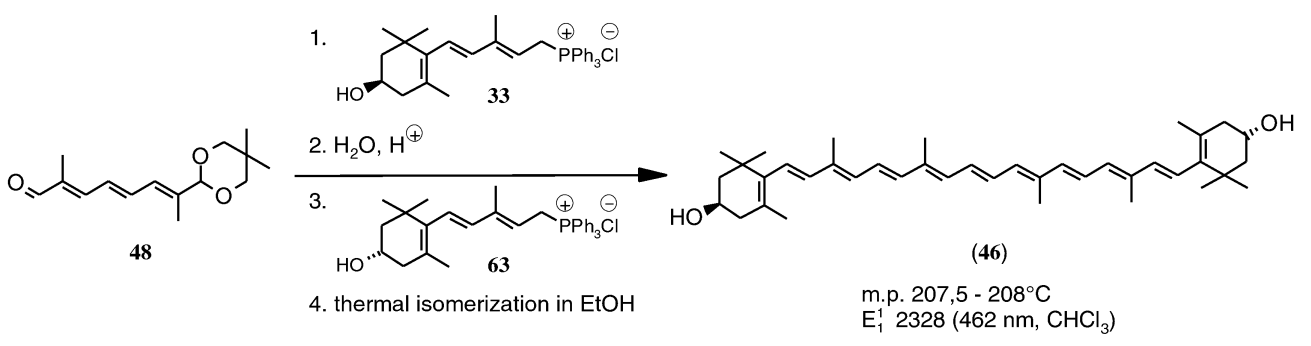

Fig. $17\left(3 R, 3^{\prime} S\right)$-meso-Zeaxanthin (46)-final steps.

\section{ACKNOWLEDGMENTS}

I wish to thank all colleagues at BASF, especially Joachim Paust and Wolfgang Krause for excellent cooperation, Daniela Klein and Martin Klatt for many fruitful discussions, and technicians Klaus Henrich, Wolfgang Kriegl, and Heike Baumann for their skillful laboratory work. My thanks also go to Kerstin Luther and Simone Bleh, who designed the layout of the manuscript.

\section{REFERENCES}

1. J. Paust. In Carotenoids, Vol. 2: Synthesis, G. Britton, S. Liaaen-Jensen, H. Pfander (Eds.), Chap. 3, Part VII, pp. 259-292, Birkhäuser, Basel (1996).

2. H. Ernst. In Carotenoids, Vol. 2: Synthesis, G. Britton, S. Liaaen-Jensen, H. Pfander (Eds.), Chap. 2, Part III, pp. 79-102, Birkhäuser, Basel (1996).

3. (a) G. A. Chasse, K. P. Chasse, A. Kucsmann, L. L. Torday, J. G. Papp. THEOCHEM 571, 7-26 (2001); (b) G. A. Chasse, L. M. Mak, E. Deretay, L. Farkas, L. L. Torday, J. G. Papp, D. S. R. Sarma, A. Agarwal, S. Chakravarthi, S. Agarwal, A. V. Rao. THEOCHEM 571, 27-37 (2001).

4. K. Meyer. Hoffmann-La Roche, Europ. Pat. 382067 (1990).

5. C. Wegner and M. John. BASF, Ger. Pat. 10009459 (2000).

6. E. Widmer. Pure Appl. Chem. 57, 741-752 (1985).

7. E. Widmer, R. Zell, E. A. Broger, Y. Crameri, H. P. Wagner, J. Dinkel, M. Schlageter, T. Lukác. Helv. Chim. Acta 64, 2436-2446 (1981).

8. H. Ernst, W. Dobler, J. Paust, U. Rheude. BASF, Europ. Pat. 633258 (1994). 
9. E. A. Broger, Y. Crameri, R. Schmid, T. Siegfried. Hoffmann-La Roche, Europ. Pat. 691325 (1995).

10. M. Scalone, R. Schmid, E. Broger, W. Burkhart, M. Cereghetti, Y. Crameri, J. Foricher, M. Hennig, F. Kienzle, F. Montavon, G. Schoettel, D. Tesauro, S. Wang, R. Zell, U. Zutter. Proceedings of the ChiraTech'97 Symposium, The Catalyst Group, Spring House, USA (1997).

11. H. Mayer. Pure Appl. Chem. 51, 535-564 (1979).

12. A. Rüttimann and H. Mayer. Helv. Chim. Acta 63, 1456-1462 (1980).

13. H. G. W. Leuenberger, W. Boguth, E. Widmer, R. Zell. Helv. Chim. Acta 59, 1832-1849 (1976).

14. J. Paust, W. Kriegl, H. Hartmann. Europ. Pat. 775685 (1996).

15. M. Fukuoka, K. Hiraga, T. Skihara. Hoffmann-La Roche, Europ. Pat. 1074630 (2000).

16. Y. Crameri, K. Puentener, M. Scalone. Hoffmann-La Roche, Europ. Pat. 915076 (1998).

17. E. Widmer, M. Soukup, R. Zell, E. Broger, H. P. Wagner, M. Imfeld. Helv. Chim. Acta 73, 861-867 (1990).

18. M. Soukup, E. Widmer, T. Lukac. Helv. Chim. Acta 73, 868-873 (1990).

19. J. Paust and W. Kriegl. BASF, Europ. Pat. 882709 (1998).

20. H. Pfander. Key to Carotenoids, Birkhäuser, Basel (1987).

21. G. A. Rodriguez. Prodemex, WO 99/03830 (1999).

22. K. Bernhard et al., Hoffmann-La Roche, Europ. Pat. 834536 (1997).

23. M. Torres-Cardona and J. Torres-Quiroga. Industrial Organica, Europ. Pat. 741795 (1995).

24. K. Bernhard and A. Rüttimann. Helv. Chim. Acta 64, 2469-2484 (1981).

25. R. K. Müller. In Carotenoids, Vol. 2: Synthesis, G. Britton, S. Liaaen-Jensen, H. Pfander (Eds.), Chap. 3, Part I, pp. 115-129, Birkhäuser, Basel (1996).

26. Semmelhack et al. J. Amer. Chem. Soc. 106, 3374-3376 (1984).

27. G. Knaus and J. Paust. BASF, Europ. Pat. 282760 (1988).

28. W. Krause and J. Paust. BASF, Europ. Pat. 718283 (1995).

29. H. Ernst. Pat. Appl. filed.

30. A. Rüttimann, K. Schiedt, M. Vecchi. J. High Res. Chromatogr. Chrom. Commun. 6, 612-616 (1989). 